\title{
RELATIVE DISTANCE: THE KEY TO THE SHAPE OF HEPATIC BUILDING BLOCKS
}

\author{
JAN M RuiJTER, MARRY M MARKMAN, JACO HAgOORT, ANTOON FM MOORMAN, Wouter H \\ LAMERS
}

Department of Anatomy and Embryology, Academic Medical Center, Meibergdreef 15, 1105 AZ, Amsterdam, The Netherlands

(Accepted January 20, 2000)

\begin{abstract}
The delineation and the shape of the smallest structural units of the liver is still the subject of debate. However, the blood flow from an upstream terminal branch of the portal vein to a downstream central vein is thought to induce a functional zonation in hepatocyte gene expression. This property was used to determine boundary conditions for the shape of the hepatic building blocks. Histochemical techniques that specifically label periportally or pericentrally expressed enzymes can be used to distinguish periportal and pericentral areas in a liver section. Pairs of images from aligned serial sections, one stained for a portal and the next for a central enzyme, are used. Segmentation and skeletonisation of these images results in the skeletons of the portal and central areas. Distance transformation with respect to these skeletons gives for each point in the image pair the distance to the nearest terminal branches of the portal vein and the central vein. For each point the relative position on the porto-central radius can then be calculated as its distance to a portal vein divided by the sum of its portal and its central distance. In the resulting relative radius image, the area occupied by 'zones' of equivalent relative radius can be measured. According to the principle of Delesse the relative area of a zone in the image is equal to the relative volume of that zone in the tissue. For structural units of plate-like, cylindrical or spherical shape, the relative volume of a zone is equal to the relative radius of that zone to the power 1,2 or 3 , respectively. Thus, the exponent in the relative area - relative radius relation gives information on the shape of the structural unit. Measurement of the areas of each relative radius zone and determination of the area - radius relation in images of random sections of adult mouse liver results in an exponent of 1.1. This suggests that the smallest structural unit of the mouse liver has the shape of a needle.
\end{abstract}

Keywords: distance transform, hepatocytes, liver lobule, stereology.

\section{INTRODUCTION}

Histologically, the structure of liver parenchyma can be described as a sponge-like structure ('muralium') composed of epithelial cells. The delineation and the shape of the smallest structural units of the liver is the subject of debate (Teutsch et al., 1999). The classic liver lobule is described as a hexagonal 'cylinder' centred around the efferent terminal branches of the hepatic vein, the so-called central veins of the lobule (Kiernan, 1833). The acinar model of liver structure, on the other hand, centres the smallest unit of the liver around the afferent terminal branches of the portal veins (Rappaport, 1954). Based on stereoangiography Matsumoto and Kawakami (1982) describe the classical cylindrical unit to consist of cone-shaped sub-structures. Microcirculatory studies also led to the description of a cone-shaped unit, the choleohepaton: a parenchymal subdivision that is serviced by one portal inlet venule (Ekataksin et al., 1997). Irrespective of their differences, all models are based on the presumed topographical distribution of the terminal branches of the portal and hepatic veins. However, except for the more or less parallel vessels that terminate on the surface of the liver (Teutsch et al., 1999), the structural basis of their branching pattern has remained elusive (Takahashi, 1970). Nevertheless, the acinar model predicts that the summed length of the terminal branches of the hepatic vein exceeds that of the terminal branches of the portal vein, whereas the prediction for the lobular model is the opposite. An unbiased estimate of these parameters could therefore discriminate between the models. The advent of specific histochemical techniques (enzyme histochemistry, immunohistochemistry and in situ hybridisation) leads to the awareness that not all hepatocytes display the same enzyme expression pattern. These observations formed the basis for the concept of the metabolic lobulus (Lamers et al., 1989). Although the exact shape of the metabolic lobulus was still unknown, this putative unit could be subdivided in a 
portal area and a central area, each with its own specific combination of enzyme activities. This functional zonation is thought to be brought about by the unidirectional flow of blood from an upstream terminal branch of the portal vein to a downstream central vein (Christoffels et al., 1999). The position of a hepatocyte along the porto-central axis of the putative liver unit determines its expression pattern (Lamers et al., 1997). A method to directly measure these porto-central gradients in gene expression has recently been developed (Ruijter et al., in preparation). This method is based on the determination of the relative position of the hepatocyte between the nearest portal and nearest central vein in the section. This measurement method also provided data to determine boundary conditions for the shape of the hepatic building blocks.

\section{STEREOLOGICAL MODEL}

The classic liver lobule can be visualised as a cylindrical structure with a central vein in the axis and portal veins around the periphery (Fig. 1A). Along the porto-central axis of this lobule several zones of enzyme expression can be distinguished, each of which will show up as a ellipse-shaped ring on a random section through the lobule (Fig. 1B). Note that all points along the inner or outer border of such a ring have the same relative position on the porto-central radius. This relative position on the porto-central radius, relative radius for short, is defined as the distance to the portal vein ( $\mathrm{p}$ ) divided by the sum of the distance to the portal and the central vein (c; Eq. 1):

$$
\text { relative radius }=\frac{p}{p+c}
$$

For a cylinder the relative volume of zone $\mathrm{X}$, from position $r_{1}$ to position $r_{2}\left(r_{0}\right.$ being the centre of the cylinder) is given by Eq. 2, in which $\mathrm{h}$ is the height of the cylinder.

$$
\text { relative volume } e_{x}=\frac{h \pi\left(r_{2}^{2}-r_{1}^{2}\right)}{h \pi r_{\text {tot }}^{2}}
$$

The cumulative relative volume, from $r_{0}$ up to and including zone $\mathrm{X}$ is then:

cumulative relative volume $\mathrm{x}_{\mathrm{x}}=\frac{\mathrm{r}_{2}^{2}}{\mathrm{r}_{\text {tot }}^{2}}=\left(\text { relative radius }{ }_{\mathrm{x}}\right)^{2}$

According to the principle of Delesse the relative volume of zone $\mathrm{X}$ in the tissue is equal to the relative area of zone $\mathrm{X}$ in a random section through the tissue (Howard and Reed, 1998). Therefore, for each zone of a cylindrical structure, effectively a stack of disk-like structural units, the cumulative relative area in a section is equal to the relative radius squared. Similarly it can be derived that for spherical structural units the exponent of this area - radius relation is 3 , whereas on the other end of the scale, for a needle-like structural element this exponent is 1 (Fig. 1C). Thus, the exponent of the cumulative relative area - relative radius relation gives information on the shape of the structural unit.
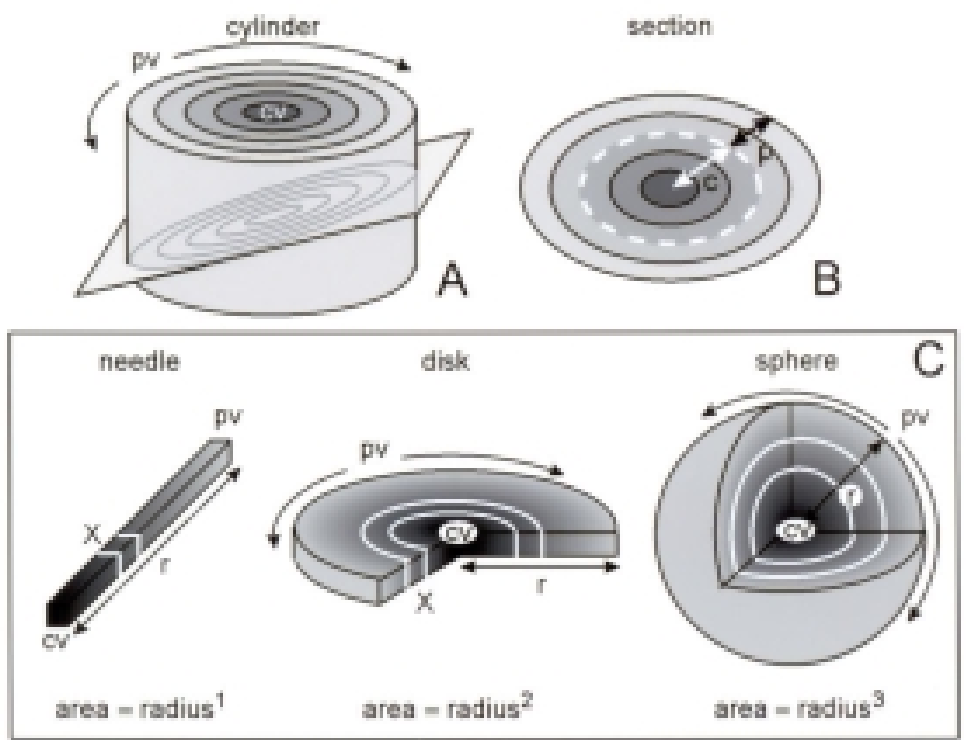

Fig. 1. Stereological model. A: cylindrical liver lobule with a random section plane, pv: portal veins, cv: central vein. B: Resulting section through the lobule, $p$ : portal distance, $c$ : central distance. The white dotted line connects points with the same relative radius $p /(p+c) . C$ : comparison of the area - radius relation in three structural units of different shape. See the text for details. 


\section{MATERIAL AND METHODS}

Material. Adult mouse liver was fixed, embedded in Paraplast and sectioned $(7 \mu \mathrm{m})$ in a random orientation. Pairs of serial sections were processed for in situ hybridisation (Moorman et al., 1999) with probes for the detection of glutamine synthetase (GS) and phosphoenolpyruvate carboxykinase (PEPCK). In images of the resulting sections the visualisation of the GS and PEPCK probes allowed the identification of central and portal areas, respectively (Figs. 2A, B).

Image acquisition. Pairs of aligned images were acquired using a Photometrics CCD camera on a Zeiss Axiophot microscope with a 5 times magnification. These images were converted into Optical Density (OD) images by calculating the negative logarithm of the transmission image (I) divided by an image of the light source $\left(\mathrm{I}_{0} ; \mathrm{OD}={ }_{-}^{10} \log \left(\mathrm{I} / \mathrm{I}_{0}\right)\right.$. The digital images were stored as 8 bits grey value images (OD value times 250) with a final format of $658 \times 517$ pixels. Each image represents $1.8 \times 1.4 \mathrm{~mm}^{2}$ of the liver section; 8 pairs of images were used per mouse.

Image processing. The image processing procedure, uses a pair of aligned OD images, specifically stained either for a portally or a centrally located enzyme. The objective of the procedure is to obtain an image in which each pixel value represents the position of that pixel on the porto-central axis (Eq. 1; Fig. 1B). An automatic segmentation procedure (Hagoort et al., 1999) was used to divide each OD image (Figs. 2A, B) into a number of continuous zones of similar OD level. This procedure uses an iterative algorithm in which each step starts with setting a relative threshold based on the histogram of the image/segment. The grey value variation of the resulting image parts is measured after a series of binary operations to fill small holes and remove small particles. Then a decision criterion, based on the pooled grey value variation of the 'daughter' segments compared to that of the 'mother' segment, serves to determine whether or not this splitting of the image was justified. This segmentation procedure results in 10-12 zones per image (Figs. 2C, D). The segmentation result is reproducible and independent of the absolute OD values in the images (Hagoort et al., 1999). The portal and central veins, which have an optical density value close to the tissue background have to be interactively marked in those images. The segmented images are then converted into binary images by accumulating portal or central zones, starting from the darkest zone and including the enclosed blood vessels, until more than $15 \%$ but less than $30 \%$ of the image area is selected (Figs. 2E, F). In the resulting binary images, the contours of the portal or central areas are smoothed by a series of binary "open" and "close" operations with increasing step size. The resulting smoothed binary images identify the portal (Fig. 2H) and central (Fig. $2 \mathrm{G}$ ) areas and skeletonisation of both images leads to images with one pixel thick portal (Fig. 2J) or central (Fig. 2I) skeletons on which the respective vessels were superimposed. The next step of the procedure is a distance transformation with respect to these skeletons. This results in images in which each pixel value represents the distance of that pixel to the nearest skeleton/vessel (Figs. 2K, L). These two distance images are then combined to obtain the relative radius image (Fig. 2M) using image arithmetic: portal distance image / (portal distance image + central distance image). In the resulting relative radius image the pixel values range between 0 (for pixels on the portal skeleton) to 1 (for pixels on the central skeleton). Portal, central and relative radius images were created as 16 bits images (relative radius times 1000).

Area measurement. In the relative radius image the area occupied by a narrow range of relative radius values is measured. For these measurements the total radius was divided into 20 steps. Measurements were done in a region of interest that excluded the outer rim of the image thus avoiding artefacts caused by the image margins (Fig. 3). The discarded rim of the image is $0.2 \mathrm{~mm}$ wide which is approximately one porto-central distance. For graphical presentation, the measured areas were accumulated starting with the most central zone and the total area was set to 1 to calculate the cumulative relative area associated with each relative radius zone.

\section{RESULTS AND DISCUSSION}

The relation found between cumulative relative area and relative radius is plotted in Fig. 4 (filled triangles). As can be seen the observed relation, with an exponent of 1.1 , closely approximates the relation $\mathrm{Y}=\mathrm{X}$, indicating that the structural unit of the adult mouse liver is shaped as a needle or a plate. When similar calculations are performed on data from sections derived from the outer $1.5 \mathrm{~mm}$ of rat liver (Teutsch et al., 1999), the exponent of the area - radius relation turns out to be about 1.7, indicating that the shape of these peripheral hepatic units is almost cylindrical (Fig. 4), which is in agreement with their three dimensional reconstruction of a liver unit. These findings show that, except for the peripheral subcapsular layer, the minimal unit of the liver is best represented by a single row of hepatocytes surrounded by sinusoids, the microvascular unit of McCuskey (1994) or by a narrow cone- or wedgeshaped unit such as the choleohepaton of Ekataksin 
(1997).

Several assumptions and decisions in the image processing and analysis procedure presented here have an influence on the result. Firstly, the result is affected by the choice of the marker enzymes to identify the portal and central areas. When enzymes with a wider distribution around the vessels are used, larger portal and central areas may result. However, the binarization step limits the portal or central zones to a maximum of $30 \%$ of the image area, which effectively neutralises the effect of the wider distribution of the enzymes. Preferably, only enzymes that display a narrow and steep expression gradient around one of the vessels should be used. The use of an automatic segmentation procedure (Hagoort et al., 1999) guarantees that the absolute staining intensity does not affect the reproducibility of the area identification.

Secondly the relative position of a point on the porto-central axis is only independent of the sectioning direction when the portal and central component of the structure are parallel. When the liver lobule is assumed to be tortuous, the deviation of the observed relative radius from the real relative radius depends on the position of the section on the arcing lobule (Fig. 5A). However, assuming that the lobules will twist and turn in all possible directions, the mean observed relative radius will never be more then $2.5 \%$ from the real relative radius when both the portal and the central end of the radius are present in the image (Fig. 5B). This measuring error, that occurs only in a very small fraction of the sections is considered to be acceptable. A more serious problem is presented by the portal and/or central veins that are missed because they are not in the plane of sectioning. In the calculation of the relative radius (Eq. 1) distances to vessels that are missed will be substituted by the distance to the next closest vessel of that type, which is in most cases a considerably longer distance. This will be strongly in favour of relative positions away from the vessels that were missed. When the length densities of portal and central veins differ, this bias will lead to exaggeration of relative positions close to the component with the highest length density and, therefore, to an area - radius relation with an exponent deviating from 1 . The fact that we find an exponent very close to 1 despite this sectioning error, indicates that both portal and central skeletons are equally affected by it. However, it may also be that the structural unit of the liver is that long and tortuous that it is almost impossible to section it without hitting both ends of the porto-central axis.

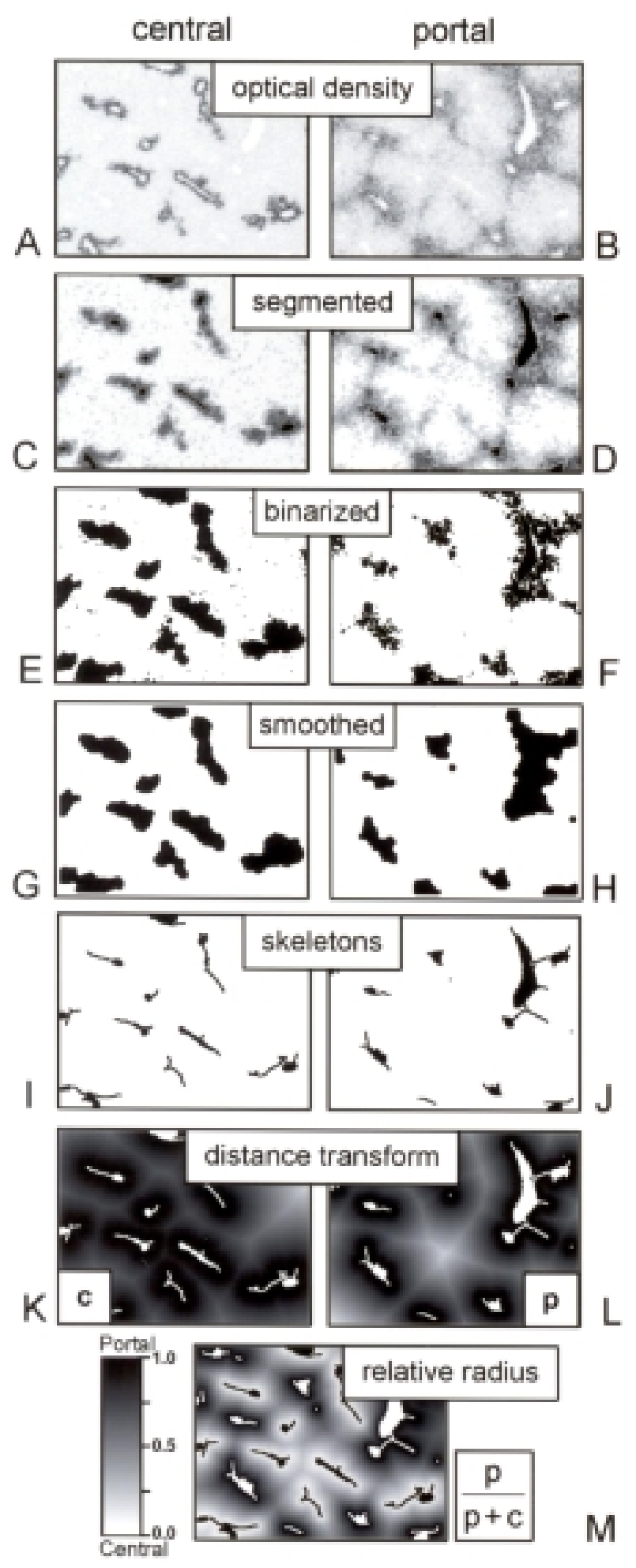

Fig. 2. Illustration of the image processing steps needed to calculate a relative radius image $(M)$ from a pair of Optical Density images (A, B). Refer to the text for a detailed description. 


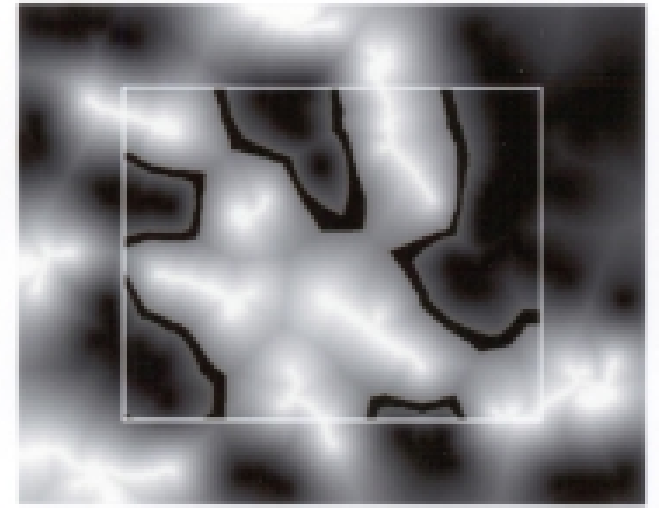

Fig. 3. Measurement of the area occupied by a narrow range of relative radius values (black zone). Only the part of the image within the white line is included in the measurement.

In conclusion, the present study indicates that the smallest structural unit of mouse liver is needle-shaped and that the amount of portal and central vein associated with each unit is equal. The functional implications of this finding for hemodynamics, blood-hepatocyte interaction and regulation of gene expression are under study.

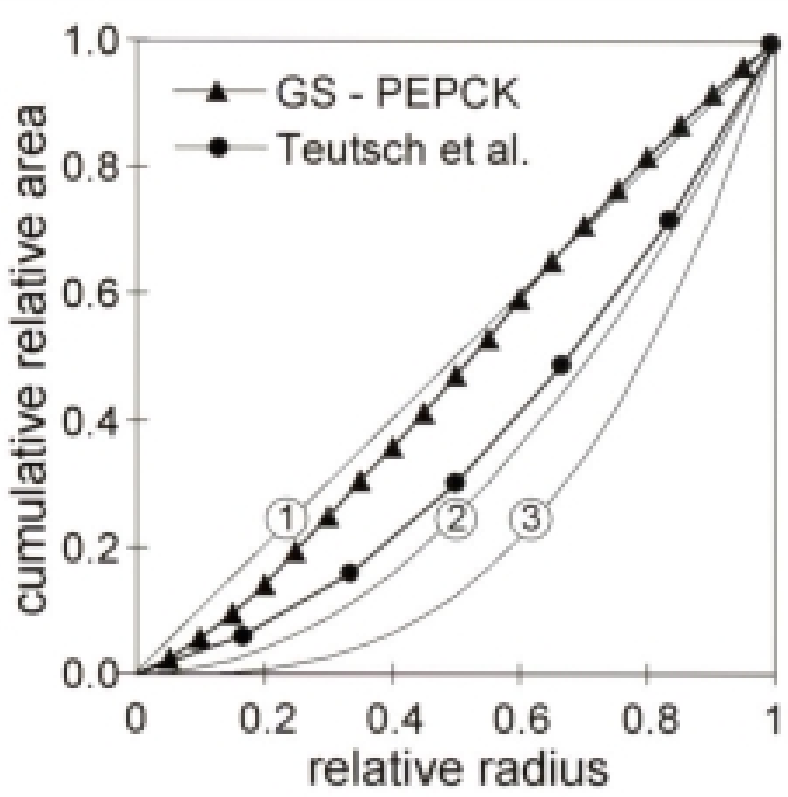

Fig. 4. Relation between the cumulative relative area and the relative radius. Filled triangles: relation based on the relative radius when PEPCK is used as the portal marker and GS as central marker in a random series of mouse liver sections. The exponent of this line when fitted to the relation area $=$ radius ${ }^{A}$ is 1.1. Filled circles: area - radius relation found for the data of Teutsch et al. (1999). The exponent of this line, based on a set of section from the outer $1.5 \mathrm{~mm}$ of rat liver is 1.7. The area - radius relations for a plate (exponent 1 ), cylinder (2) and sphere (3) area added for comparison.

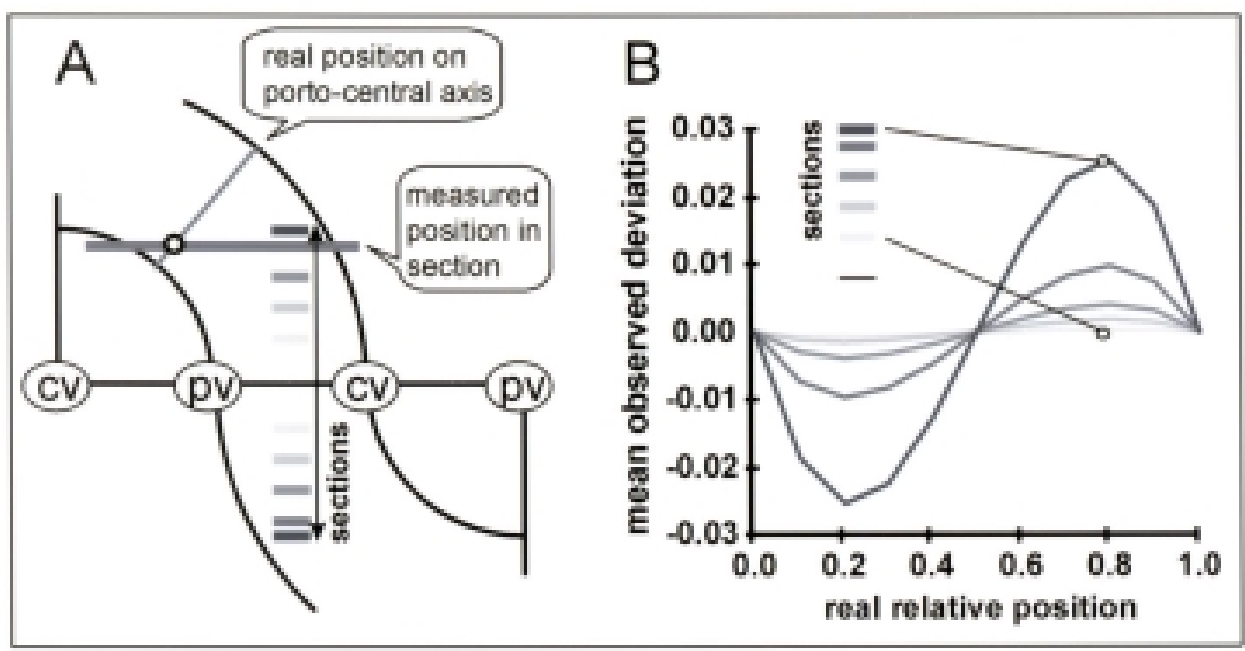

Fig. 5. Simulation of the error in the determination of the relative position when the portal and central poles of the structural unit are not parallel. A: schematic drawing through a maximally curved lobule, pv: portal vein, $c v$ : central vein. The positions of a selection of 'sections' that hit a portal as well as a central vein are indicated. For each point in such a section the observed and the real relative position can be calculated. B: mean observed deviation ((observed position - real position)/real position) as a function of the real position for the sections indicated in A. Note that 90\% of the possible sections result in a measurement error of less than $1 \%$. 
A preliminary report of some of the data (Ruijter et al., 1999) has been presented at the $X^{\text {th }}$ International Congress for Stereology, Melbourne, Australia, 1-4 November 1999.

\section{ACKNOWLEDGEMENT}

The authors wish to thank Drs Vincent Christoffels, Roben Gieling and Gerry Wagenaar for their contributions to this study.

\section{REFERENCES}

Christoffels VM, Sassi H, Ruijter JM, Moorman AFM, Grange T, Lamers WH (1999). A mechanistic model for the development and maintenance of portocentral gradients in gene expression in the liver. Hepatology 29:1180-92.

Hagoort J, Salam K, Ruijter JM (1999). Automatic segmentation of optical density images. Acta Stereol 18:265-74.

Ekataksin W, Zou Z, Wake K, Chunhabundit P, Somana R, Nishida J, McCusky RS (1997). The hepatic microcirculatory subunits: an over-three-century-long search for the missing link between an exocrine unit and an endocrine unit in mammalian liver lobules. In: Motta PM, Ed. Recent advances in microscopy of cells, tissues and organs. Rome: A. Delphino Editore, 406-12.

Howard CV, Reed MG (1998). Unbiased stereology. Threedimensional measurement in microscopy. Bios Scientific Publishers Ltd. Oxford, 55-68.

Kiernan F (1833). The anatomy and physiology of the liver. Philos Tr Roy Soc Lond 123:711-70.
Lamers WH, Moorman AFM, Charles R (1989). The metabolic lobulus, a key to the architecture of the liver. Cell Biology Reviews 19:5-41.

Lamers WH, Geerts WJC Jonker A, Verbeek FJ, Wagenaar GTM, Moorman AFM (1997). Quantitative graphical description of portocentral gradients in hepatic gene expression by image analysis. Hepatology 26:398-406.

Matsumoto T, Kawakami M (1982). The unit concept of hepatic parenchyma. A re-examination based on angioarchitectural studies. Acta Pathol Jpn 32(suppl 2):285-314.

McCuskey RS (1994). The hepatic microvascular system. In: Arias IM, Boyer JL, Fausto N, Jakoby WB, Schachter DA, Shafritz DA eds. The liver: biology and pathobiology. 3rd edn. New York: Raven Press, Ltd.

Moorman AFM, De Boer PAJ, Ruijter JM, Hagoort J, Franco D, Lamers W (1999). Radio-isotopic in situ hybridisation on tissue sections: practical aspects and quantification. In: Tuan RS, Lo CW, Eds. Methods in Molecular Biology, 137 Developmental Biology Protocols, Vol III.

Rappaport AM, Borowy ZJ, Lougheed WM, Lotto N (1954). Subdivision of hexagonal liver lobules into a structural and functional unit. Anat Rec 119:11-34.

Ruijter JM, Hagoort J, Markman MMW, Gieling RG, Moorman AFM, Lamers WH. Zonal gene expression and the shape of the structural unit in the liver: approached from a distance. In preparation.

Takahashi T (1970). Lobular structure of the human liver from the viewpoint of hepatic vascular architecture. Tohoku J Exp Med 101:119-40.

Teutsch HF, Schuerfeld D, Groezinger E (1999). Threedimensional reconstruction of parenchymal units in the liver of the rat. Hepatology 29:494-505. 